\title{
Fatores associados ao LDL-Colesterol aumentado na população adulta brasileira: Pesquisa Nacional de Saúde
}

\author{
Factors associated with high LDL-Cholesterol in the Brazilian \\ adult population: National Health Survey
}

Ana Carolina Micheletti Gomide Nogueira de Sá (https://orcid.org/0000-0002-0122-2727) ${ }^{1}$

Ísis Eloah Machado (https://orcid.org/0000-0002-4678-2074) ${ }^{2}$

Regina Tomie Ivata Bernal (https://orcid.org/0000-0002-7917-3857) ${ }^{1}$

Deborah Carvalho Malta (https://orcid.org/0000-0002-8214-5734) ${ }^{3}$

${ }^{1}$ Programa de PósGraduação em Enfermagem, Escola de Enfermagem, Universidade Federal de Minas Gerais (UFMG). Av Alfredo Balena 190, Santa Efigênia. 30130-100 Belo Horizonte MG Brasil. carolmichelettigomide@ gmail.com

${ }^{2}$ Departamento de Medicina de Família, Saúde Mental e Coletiva, Escola de Medicina, Universidade Federal de Ouro Preto. Ouro Preto MG Brasil.

${ }^{3}$ Departamento de Enfermagem MaternoInfantil e Saúde Pública, Escola de Enfermagem, UFMG. Belo Horizonte MG Brasil.

\begin{abstract}
The study analyzed factors associated with high LDL-Cholesterol in Brazilian population. This is a cross-sectional study with laboratory data from 8,534 individuals collected in National Health Survey were analyzed. The prevalence levels of LDL-Cholesterol $<130$ and $\geq 130 \mathrm{mg} / \mathrm{dL}$ were calculated. The outcome variable was high LDL-Cholesterol $(\geq 130 \mathrm{mg}$ ) $d L)$ and explanatory variables were sociodemographic, anthropometric, lifestyle, chronic diseases and self-rated health. To Poisson regression was used and estimated prevalence ratios $(P R)$ with $95 \%$ confidence levels (CI) to verify associations. The prevalence of high LDL-Cholesterol was $18.58 \%$. In the final multivariate model were associated with the outcome: 30 to 44 years (PR 1.99; CI 1.58-2.54), 45 to 59 years (PR 2.89; CI 2.29-3.64), 60 years or more ( $P R$ 2.90; CI 2.29-3.68), living in the Northeast Region (PR 1.16; CI 1.02 - 1.32), overweight (PR 1.32; CI 1.15-1.51), obesity (PR 1.41; CI 1.19-1.65) or anemia (PR 0.66; CI 0.54-0.80). The LDL-Cholesterol was associated with aging, overweight, obesity, live in the Northeast and anemia. The monitoring of LDL levels is relevant, due to the increased risk with age, and can guide the adopting healthy lifestyles and diagnosis in places with lower access.

Key words Cholesterol, LDL, Dyslipidemias, Health surveys, Risk factors, Laboratory test
\end{abstract}

Resumo $O$ estudo analisou os fatores associados ao LDL-Colesterol aumentado na população adulta brasileira. Estudo transversal com dados laboratoriais de 8.534 indivíduos coletados na Pesquisa Nacional de Saúde. Calculadas as prevalências de LDL-Colesterol $<130$ e $\geq 130 \mathrm{mg} /$ $d L$. A variável desfecho foi LDL-Colesterol aumentado $(\geq 130 \mathrm{mg} / \mathrm{dL})$ e as variáveis explicativas foram sociodemográficas, antropométricas, estilo de vida, doenças crônicas e autoavaliação de saúde. Para verificar as associações, utilizouse regressão de Poisson e estimou-se as razões de prevalência $(R P)$ e intervalos de confiança (IC) 95\%. A prevalência de LDL-Colesterol aumentado foi $18,58 \%$. No modelo final multivariado associaram-se ao desfecho: idade entre 30 a 44 anos (RP 1,99; IC 1,58-2,54), 45 a 59 anos ( $R P$ 2,89; IC 2,29-3,64) e 60 anos ou mais (RP 2,90; IC 2,29-3,68), região Nordeste ( $R P$ 1,16; IC 1,02-1,32), sobrepeso (RP 1,32; IC 1,15-1,51), obesidade (RP 1,41; IC 1,19-1,65) ou anemia (RP 0,66; IC 0,54-0,80). O LDL-Colesterol aumentado associou-se ao envelhecimento, sobrepeso, obesidade, morar na região Nordeste e ter anemia. Monitorar os níveis de LDL é importante, pelo risco aumentado com envelhecimento, para orientar ações de estilos de vida saudáveis e diagnóstico em locais de menor acesso.

Palavras-chave LDL-Colesterol, Dislipidemias, Inquéritos epidemiológicos, Fatores de risco, Testes laboratoriais 


\section{Introdução}

A população adulta, no Brasil e em outros países, está exposta às condições de adoecimento decorrentes de níveis altos de colesterol. Na década de 1960, foram apresentadas evidências de que valores elevados de colesterol sérico aumentavam o risco de infarto agudo do miocárdio (IAM) ${ }^{1}$; desde então, pesquisas confirmaram a associação entre os níveis altos de colesterol, não apenas com risco de IAM, mas também de doenças arteriais periféricas e acidentes vasculares ${ }^{2}$.

As concentrações anormais de lipídios, ou lipoproteínas, circulantes na corrente sanguínea, especialmente o colesterol e os triglicerídeos (TG), são definidas como dislipidemias, as quais cursam com ou sem repercussão sobre o território vascular, associadas a outras manifestações clínicas que podem aumentar o risco de doenças cardiovasculares $^{3,4}$. As dislipidemias podem ser resultados de anormalidades genéticas, doença subjacente ou decorrentes de fatores ambientais ${ }^{5}$.

Sabe-se que a elevação dos níveis do colesterol lipoproteína de baixa densidade (LDL-Colesterol) é um dos principais contribuintes para doenças cardiovasculares (DCV) ateroscleróticas ${ }^{6,7}$. As lipoproteínas são responsáveis pelo transporte e pela solubilização dos lípides. São compostas por lípides e proteínas denominadas Apolipoproteínas (Apo). Uma das classes de lipoproteínas é rica em colesterol e inclui as lipoproteínas de baixa densidade (LDL), que é a principal transportadora de colesterol na circulação para os tecidos periféricos ${ }^{8}$. A LDL está diretamente relacionada à patogênese da aterosclerose, que é considerada a base da maioria dos eventos cardiovasculares. Dessa forma, essa lipoproteína é apontada como o melhor preditor de risco cardíaco ${ }^{2}$ e tem sido alvo terapêutico para reduzir os riscos de $\mathrm{DCV}^{10}$.

O último relatório da Organização Mundial de Saúde (OMS), em 2009, mostrou que os níveis aumentados de colesterol sérico causaram 2,6 milhões de mortes e 29,7 milhões de anos de vida perdidos por morte prematura e incapacidades ${ }^{11}$. Especificamente para os riscos atribuídos ao LDL-Colesterol aumentado, em 2017, estimativas apontaram a ocorrência de 4,3 milhões de mortes globais, correspondendo a 7,7\% de todos os óbitos e 94,9 milhões de anos de vida perdidos ajustados por incapacidade (DALYS) ${ }^{12,13}$. No Brasil, também evidencia-se a expressiva carga de morte e incapacidade atribuída ao LDL-Colesterol aumentado, apenas em 2017 estimou-se a ocorrência de 99.315 óbitos $(7,36 \%$ do total de mortes) e a perda de 2.335.294,99 DALYS ${ }^{13}$.
Esse cenário mostra a importância de políticas públicas direcionadas ao controle de fatores modificáveis como as dislipidemias. Sobretudo como estratégia para a redução da morbimortalidade por DCV, consideradas as principais causas de morte no mundo ${ }^{9,14-18}$.

Nessa perspectiva, o conhecimento das dislipidemias por meio da análise do perfil lipídico é fundamental, haja vista que visa a identificar alterações isoladas ou combinadas ${ }^{5,15}$ dos níveis de colesterol total (CT), TG, fração de LDL-Colesterol e fração de colesterol da lipoproteína de alta densidade (HDL-Colesterol) ${ }^{14}$.

Quanto aos fatores associados às dislipidemias, são apontados os sociodemográficos, como sexo, idade ${ }^{15,19-21}$, escolaridade ${ }^{22}$, local de residência $^{15,19,20}$, estilos de vida ${ }^{19-21}$, sobrepeso, obesida$\mathrm{de}^{15,16,19,20}$, doenças crônicas, como hipertensão e diabetes $^{15,20}$, e autoavaliação de saúde ruim ${ }^{19}$.

No Brasil, os inquéritos epidemiológicos que monitoram as prevalências de dislipidemia são escassos e, na maioria das vezes, utilizam dados autorreferidos ${ }^{19,23}$. Nesse contexto, visando o monitoramento dos indicadores de risco para Doenças Crônicas Não Transmissíveis (DCNT), a Pesquisa Nacional de Saúde (PNS) realizou coletas de material biológico que incluíram medidas de colesterol e frações ${ }^{24-26}$ e permitiu monitorar as dislipidemias na população brasileira por meio de exames laboratoriais ${ }^{26}$.

Assim, considerando-se a relevância supracitada das repercussões negativas na saúde, ocasionadas por aumento dos níveis de LDL-Colesterol e que, por isso, na prática clínica, essas estruturas moleculares circulantes têm tido tanta importân$\mathrm{cia}^{27}$, torna-se necessária a realização de estudos que investiguem os fatores associados ao LDLColesterol aumentado no Brasil.

Nesse sentido, este estudo inovou ao fazer análises inéditas sobre fatores associados ao LDL-Colesterol aumentado nos adultos brasileiros, pelos exames laboratoriais do mais amplo inquérito de saúde do Brasil, a $\mathrm{PNS}^{28}$. Outrossim, a pesquisa avança ao trazer novidades, em termos de informações relevantes quanto ao diagnóstico populacional de LDL-Colesterol aumentado e os seus fatores associados, diante dos desafios em termos de saúde pública para a detecção do risco cardiovascular e prevenção de DCV. Ademais, este trabalho contribui como subsídio para ações de prevenção de dislipidemias, bem como para melhorar a situação de saúde da população brasileira.

Desta forma, o presente estudo teve por objetivo analisar os fatores associados à distribui- 
ção de LDL-Colesterol aumentado na população adulta brasileira.

\section{Métodos}

Trata-se de estudo transversal que utilizou a base de dados dos exames laboratoriais da PNS entre os anos de 2014 e 2015.

A PNS é uma pesquisa de âmbito nacional e base domiciliar realizada pelo Instituto Brasileiro de Geografia e Estatística (IBGE), em parceria com o Ministério da Saúde (MS). A pesquisa utilizou amostra probabilística em três estágios em que foram obtidos registros de entrevistas em 64.348 domicílios $^{24,25}$ e, para a realização dos exames laboratoriais da PNS, foi planejada a coleta de material biológico em subamostra de $25 \%$ dos setores censitários pesquisados ${ }^{24-26}$.

As análises laboratoriais da PNS foram selecionadas em uma subamostra constituída de 8.952 indivíduos. Foram excluídas 418 amostras devido a material insuficiente, hemólise e perda da amostra. Para esta análise, totalizaram-se 8.534 amostras de sangue dos indivíduos selecionados. Em função das perdas e visando a reduzir viés de representação, o estudo adotou pesos de pós-estratificação segundo sexo, idade, escolaridade e região, com o intuito de estabelecer estimativas para a população adulta brasileira ${ }^{25}$. As coletas de sangue periférico foram realizadas a qualquer hora do dia $^{26}$, e o estudo seguiu o protocolo que dispensa o jejum para a medição do colesterol ${ }^{8}$. As amostras de LDL-Colesterol foram coletadas em tubos com gel. Aguardaram-se 30 minutos para a retração do coágulo e após foi realizada a centrifugação e o encaminhamento das amostras sob refrigeração de 2 a $8^{\circ} \mathrm{C}$, com controle da temperatura nas etapas. Esse parâmetro foi dosado por método enzimático/colorimétrico automatizado ${ }^{26}$. Maiores detalhes metodológicos e da coleta laboratorial da PNS estão disponíveis em outras publicações ${ }^{24-26}$.

Este estudo incluiu variáveis referentes à fração de LDL-Colesterol (dosado nos exames laboratoriais), sociodemográficas, estilos de vida e doenças crônicas ${ }^{29}$.

A variável desfecho foi ter ou não LDL-Colesterol aumentado; para tanto, realizou-se a análise dicotômica, definida pelo ponto de corte de $\mathrm{LDL} \geq 130 \mathrm{mg} / \mathrm{dL}$, conforme recomendações do National Cholesterol Education Program (NCEP -ATPIII) ${ }^{30}$.

As variáveis explicativas foram:

a) Sociodemográficas: sexo (masculino e feminino); idade (adultos com idade maior ou igual a 18 anos); escolaridade (categorizada em analfabeto e ensino fundamental incompleto, ensino fundamental completo e médio incompleto, ensino médio completo e mais); cor da pele (branca e outras que corresponderam à amarela e indígena; preta e parda); regiões do Brasil (Norte, Nordeste, Sudeste, Sul e Centro-Oeste).

b) Antropométricas: índice de massa corporal (IMC), que foi calculado a partir de medidas de peso e altura aferidos na $\mathrm{PNS}^{28}$. O IMC foi classificado de acordo com a Organização Mundial da Saúde (OMS), como normal/baixo peso (IMC $<25 \mathrm{~kg} / \mathrm{m}^{2}$ ), sobrepeso (IMC entre 25 e 29 $\mathrm{kg} / \mathrm{m}^{2}$ ) e obesidade $\left(\mathrm{IMC} \geq 30 \mathrm{~kg} / \mathrm{m}^{2}\right)^{31}$.

c) Estilo de vida: consumo de carne vermelha com gordura. Para a construção desse indicador, utilizou-se a questão: "Quando o(a) sr.(a) come carne vermelha, o(a) sr.(a)costuma tirar o excesso de gordura visível ou comer com gordura?" Categorizaram-se respostas com "sim" e "não" para o relato de consumo de carne vermelha com gordura visível; o consumo de bebida alcóolica foi avaliado pelas questões: "Quantos dias por semana o(a) sr.(a) costuma tomar alguma bebida alcoólica?"; "Em geral, no dia em que o(a) sr.(a) bebe, quantas doses de bebida alcoólica o(a) sr.(a) consome?" (1 dose de bebida alcoólica equivale a 1 lata de cerveja, 1 taça de vinho ou 1 dose de cachaça, whisky ou qualquer outra bebida alcoólica destilada). A construção desse indicador foi embasada no consumo abusivo e frequente de álcool; para tanto, utilizou-se o conceito de heavy drinking, proposto pelo $\mathrm{CDC}^{32}$. Para esse estudo, o consumo foi classicado em: não consome (nunca ou menos de uma vez por semana), leve/ moderado (1 a 7 doses/semana para mulheres e 1 a 14 doses/semana homens ${ }^{32}$ ) e abusivo (ingestão semanal igual ou superior de 8 doses para mulheres e 15 para homens ${ }^{32}$ ); tabagismo, para a construção desse indicador, utilizou-se a questão: Atualmente, o(a) sr.(a) fuma algum produto do tabaco? Como opções de respostas, foram adotadas as seguintes construções: "Sim, diariamente"; "Sim, menos que diariamente"; "Não fumo atualmente". Foi considerado como fumante quem respondeu positivamente à questão.

d) Doenças crônicas não transmissíveis (DCNT): insuficiência renal. Esse indicador foi calculado por dados de exames laboratoriais da PNS. Considerou-se a taxa de filtração glomerular (TFG) menor que $60 \mathrm{~mL} / \mathrm{min} / 1,73 \mathrm{~m}^{2}$ no exame de sangue e, para o cálculo, foi utilizada a equação Chronic Kidney Disease Epidemiology Collaboration (CKD-EPI) ${ }^{33}$. Neste estudo não foi empregada a correção por raça conforme 
preconizado pela maioria dos métodos ${ }^{33}$. Diabetes, para a construção dessa variável utilizou-se o valor de hemoglobina glicada $\geq 6,5 \%{ }^{34,35}$ dosada por exame de sangue na PNS e o diagnóstico autorreferido da doença, considerando-se a resposta positiva para a questão: "Algum médico já lhe deu o diagnóstico de diabetes?" (Categorizada, como "sim" ou "não"). Hipertensão arterial, para a confecção desse indicador, utilizou-se o diagnóstico autorreferido para a doença, avaliado pelas questões: "Algum médico já lhe deu o diagnóstico de hipertensão arterial - pressão alta? (Categorizada como "sim" ou "não"); "Nas duas últimas semanas, $\mathrm{o}$ (a) sr.(a) tomou medicamentos por causa da hipertensão arterial (pressão alta)?" (Categorizada como "sim" ou "não"). Também utilizaram-se medidas de pressão arterial aferidas na PNS, definindo-se como hipertensão arterial, pressão sistólica $\geq 140$ e diastólica $\geq 90 \mathrm{mmHg}$, segundo os critérios da $7^{\mathrm{a}}$ Diretriz Brasileira de Hipertensão Arterial ${ }^{36}$.

e) Autoavaliação de saúde: para a construção desse indicador, utilizou-se a questão: "Em geral, como o(a) sr.(a) avalia a sua saúde?" Categorizada como "muito bom"/“bom", "regular" e "muito ruim"/"ruim".

Além disso, foram avaliadas as variáveis sociodemográficas, sexo, idade, escolaridade, cor da pele e região como possíveis fatores de confusão.

A análise descritiva das variáveis explicativas e as prevalências de LDL-Colesterol $<130 \mathrm{mg} /$ $\mathrm{dL}$ (limiar ótimo) ${ }^{31} \mathrm{e} \geq 130 \mathrm{mg} / \mathrm{dL}$ (aumentado), foram apresentadas em proporções (\%) com intervalos de confiança de 95\% (IC95\%). Para os fatores associados em relação ao desfecho, a análise considerou os blocos de determinação causal propostos pelo modelo teórico de Bergmann et al. ${ }^{37}$ e outros estudos ${ }^{15,16,19-21}$. A escolha do modelo teórico ${ }^{37}$ justifica-se por ser um estudo nacional em que os autores construíram o modelo, mas também foram contempladas outras pesquisas com adultos ${ }^{15,19-21}$ que investigaram todas as variáveis presentes neste estudo.

A entrada de cada uma das variáveis explicativas ocorreu conforme o modelo teórico hierarquizado, que considerou cinco blocos de determinação causal (Figura 1).

As análises de associações foram examinadas por meio das razões de prevalência (RP) e respectivos IC95\% e foram calculadas pelo modelo de regressão de Poisson com variância robusta. As RP acima de 1 indicam fator de risco, e as RP abaixo de 1 indicam fator de proteção.

Foram construídos três modelos de regressão. Consideraram-se, no modelo 1 , as análises
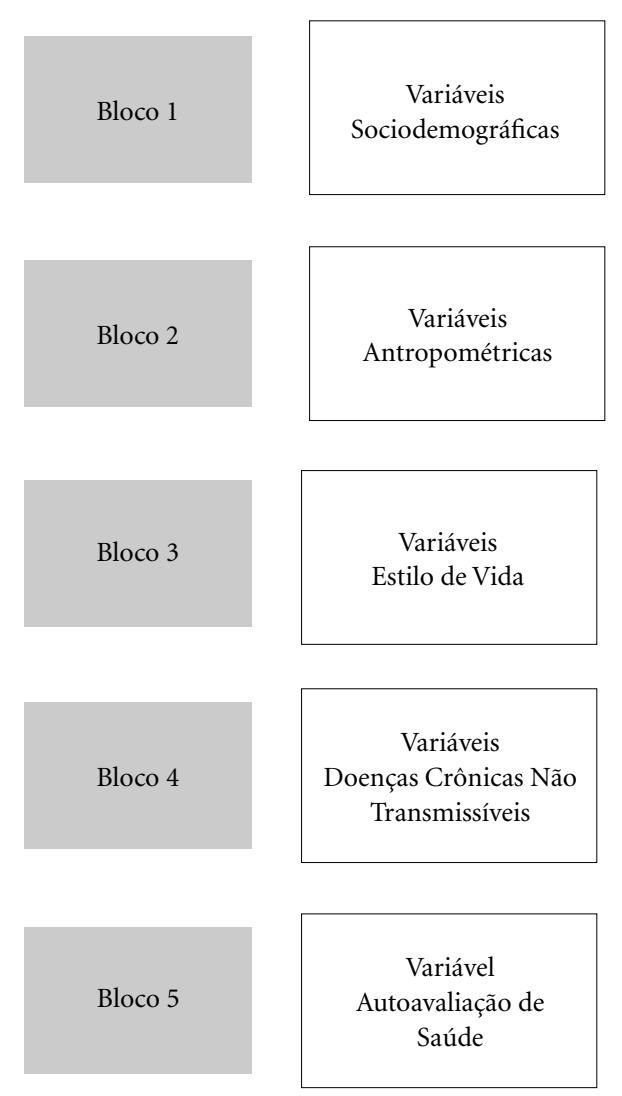

Variável Desfecho LDL-Colesterol $\geq 130 \mathrm{mg} / \mathrm{dL}$

Figura 1. Modelo teórico hierarquizado de fatores associados ao LDL-Colesterol aumentado.

bivariadas entre a variável desfecho e cada variável explicativa e estimadas as RP brutas ( $\mathrm{RPb})$. No modelo 2, foi realizada a análise ajustada por sexo, escolaridade, cor da pele e região e calculadas as RP ajustadas $\left(\mathrm{RPaj}^{\mathrm{a}}\right)$; foi excluída a variável idade, por apontar que, utilizando-se este ajuste, algumas variáveis aceitas pela comunidade científica como fatores associados às dislipidemias ${ }^{19-22}$ perderam a significância estatística. No modelo 3 (modelo final), procedeu-se a análise multivariada ajustada por todas as variáveis explicativas, foram selecionadas e incluídas no modelo as variáveis que apresentaram valor $\mathrm{p}<0,20$ nas análises bivariadas. Nesta análise, foram estimadas as 
$\mathrm{RPaj}^{\mathrm{b}}$ e considerou-se como fatores associados ao LDL-Colesterol aumentado as variáveis explicativas que apresentaram valor de $\mathrm{p} \leq 0,05$. Foram testadas variáveis de confundimento levando-se em conta os aspectos da literatura ${ }^{8,15,16,19-21,38-42}$.

Para todas as análises, consideraram-se a estrutura amostral e os pesos de pós- estratificação. As análises dos dados foram realizadas no software Data Analysis and Statistical Software (Stata), versão 14, utilizando-se o conjunto de comandos para análise de dados de inquéritos com amostra complexa (survey).

A base de dados do laboratório e os questionários da PNS encontram-se disponíveis no site do PNS da Fundação Oswaldo Cruz (www. pns.fiocruz.br). A PNS foi aprovada pela Comissão Nacional de Ética em Pesquisa (CONEP) do Conselho Nacional de Saúde (CNS), MS. A participação do adulto na pesquisa foi voluntária; e a confidencialidade das informações, garantidas. Os indivíduos selecionados assinaram o Termo de Consentimento Livre e Esclarecido e tiveram orientações sobre o recebimento do laudo contendo os resultados dos exames ${ }^{28}$.

\section{Resultados}

Na população adulta, a prevalência de LDL-Colesterol aumentado foi de 18,58\%, sendo maior nos indivíduos a partir de 30 anos (17,55\%), chegando a aproximadamente $25 \%$ naqueles com 60 anos ou mais, nos menos escolarizados, como os analfabetos e os com ensino fundamental incompleto $(21,53 \%)$, nos que apresentaram sobrepeso $(21,10 \%)$, obesidade $(23,30 \%)$, hipertensão arterial $(23,27 \%)$, diabetes $(24,72 \%)$ e nos que avaliaram sua saúde como muito ruim/ ruim $(24,24 \%)$ e regular $(22,05 \%)$. Indivíduos com anemia apresentaram menor prevalência de LDL-Colesterol aumentado (13,29\%) (Tabela 1).

$\mathrm{Na}$ Tabela 2, foram analisados os fatores associados ao LDL-Colesterol aumentado. Os resultados apresentados mostram que a magnitude das associações variou conforme os fatores analisados.

$\mathrm{Na}$ análise bivariada (modelo 1) foram fatores protetores: escolaridade, ensino fundamental completo e médio incompleto $(\mathrm{RPb}=0,78$; IC95\% 0,66-0,93); médio completo e mais ( $\mathrm{RPb}$ $=0,77$ IC95\% 0,68-0,88); cor da pele preta e parda $(\mathrm{RPb}=0,87$; IC95\% 0,77-0,97) e anemia $(\mathrm{RPb}=0,69 ; \mathrm{IC} 95 \% 0,56-0,83)$. Quanto aos fatores de risco, foram: sexo feminino $(\mathrm{RPb}$ = 1,16; IC95\% 1,03-1,30); idade, 30 a 44 anos $(\mathrm{RPb}=1,99 ; \mathrm{IC} 95 \% 1,60-2,49), 45$ a 59 anos
$(\mathrm{RPb}=2,90 ; \mathrm{IC} 95 \%$ 2,34-3,59), 60 anos e mais $(\mathrm{RPb}=2,78$; IC95\% 2,24-3,46); regiões: Nordeste $(\mathrm{RPb}=1,22 ; \mathrm{IC} 95 \% 1,08-1,38)$ e Sul $(\mathrm{RPb}=$ 1,23; IC95\% 1,05-1,45); sobrepeso ( $\mathrm{RPb}=1,53$; IC95\% 1,34-1,75) e obesidade $(\mathrm{RPb}=1,69$; IC95\% 1,45-1,96); hipertensão arterial ( $\mathrm{RPb}=$ 1,34; IC95\% 1,18-1,51), insuficiência renal ( $\mathrm{RPb}$ $=1,24 ; \mathrm{IC} 95 \% 1,01-1,51)$ e diabetes $(\mathrm{RPb}=1,38$; IC95\% 1,18-1,61); autoavaliação de saúde regu$\operatorname{lar}(\mathrm{RPb}=1,33$; IC95\% 1,18-1,50) e muito ruim/ ruim $(\mathrm{RPb}=1,47$; IC95\% 1,22-1,76). As demais variáveis como: o consumo de carne vermelha com gordura ( $p=0,433)$, consumo de bebida alcoólica leve/moderado $(\mathrm{p}=0,501)$ e abusivo $(\mathrm{p}$ $=0,154)$, bem como tabagismo $(\mathrm{p}=0,218)$ não foram significativas.

No modelo 2, na análise ajustada por sexo, escolaridade, cor de pele e região, mantiveram-se todas as associações encontradas no modelo 1, , com exceção de insuficiência renal $\left(\mathrm{RPaj}^{\mathrm{a}}=1,11\right.$; IC95\% 0,91 - 1,37) e a região Sul $\left(\mathrm{RPaj}^{\mathrm{a}}=1,21\right.$; IC95\% 0,94 - 1,33), que perderam a significância estatística.

A Tabela 3, apresenta o modelo 3. Na análise de regressão multivariada ao serem analisadas em conjunto, algumas das variáveis perderam a significância estatística e não compuseram o modelo final. Os fatores associados ao LDL-Colesterol aumentado foram: a anemia, protetora em relação ao desfecho $\left(\mathrm{RPaj}^{\mathrm{b}}=0,66\right.$; IC95\% 0,54-0,80); e entre os fatores de risco, mantiveram-se: o aumento da idade 30 a 44 anos RPaj ${ }^{\text {b }}$ $=1,99\left(\right.$ IC95\% 1,58-2,54), 45 a 59 anos $\mathrm{RPaj}^{\mathrm{b}}=$ 2,89 (IC95\% 2,29-3,64), 60 anos e mais RPaj ${ }^{\mathrm{b}}=$ 2,90 (IC95\% 2,29-3,68), região Nordeste ( $\mathrm{RPa}$ $\mathrm{j}^{\mathrm{b}}=1,16$; IC95\% 1,02-1,32), sobrepeso (RPaj ${ }^{\mathrm{b}}$ $=1,32 ;$ IC95\%1,15-1,51) e obesidade $\left(\mathrm{RPaj}^{\mathrm{b}}=\right.$ 1,41; IC95\% 1,19-1,65).

\section{Discussão}

O LDL-Colesterol medido por análise laboratorial na PNS mostrou-se elevado em um quinto da população adulta brasileira. Foram fatores associados ao LDL-Colesterol aumentado, no modelo 2, sexo feminino, faixa etária após 30 anos, escolaridade, cor da pele preta e parda, região Nordeste, IMC alterado, hipertensão arterial, diabetes e anemia. Já no modelo final, os fatores associados ao desfecho foram a idade acima de 30 anos, sobrepeso e obesidade, residir na região Nordeste e anemia. Neste estudo, os fatores associados ao LDL-Colesterol aumentado estão em conformidade com a literatura ${ }^{15,19-21,37}$. 
Tabela 1. Prevalência de LDL-Colesterol limiar ótimo $(<130 \mathrm{mg} / \mathrm{dL})$ e aumentado $(\geq 130 \mathrm{mg} / \mathrm{dL})$ e IC95\% em adultos ( $\geq 18$ anos), segundo variáveis sociodemográficas, antropométricas, estilo de vida, doenças crônicas não transmissíveis e autoavaliação de saúde. Pesquisa Nacional de Saúde, Brasil, 2014-2015.

\begin{tabular}{|c|c|c|c|c|c|}
\hline \multicolumn{2}{|l|}{ LDL-Colesterol } & \multicolumn{3}{|c|}{ Limiar ótimo } & \multirow{2}{*}{$\begin{array}{r}\text { Aumentado } \\
\text { IC } 95 \%\end{array}$} \\
\hline Variáveis & $\mathbf{n}^{\star}$ & $\%$ & IC 95\% & $\%$ & \\
\hline Total & 8.534 & 81,42 & $80,34-82,45$ & 18,58 & $17,55-19,66$ \\
\hline Sexo & 8.534 & & & & \\
\hline Masculino & & 82,87 & $81,24-84,38$ & 17,13 & $15,62-18,76$ \\
\hline Feminino & & 80,13 & $78,67-81,52$ & 19,87 & $18,48-21,33$ \\
\hline Faixa etária & 8.534 & & & & \\
\hline 18 a 29 & & 91,19 & $89,33-92,75$ & 8,81 & $7,25-10,67$ \\
\hline 30 a 44 & & 82,45 & $80,43-84,30$ & 17,55 & $15,70-19,55$ \\
\hline 45 a 59 & & 74,45 & $72,10-76,67$ & 25,55 & $23,33-27,90$ \\
\hline 60 anos ou mais & & 75,49 & $72,97-77,84$ & 24,51 & $22,16-27,03$ \\
\hline Escolaridade & 8.534 & & & & \\
\hline Analfabeto / Fundamental incompleto & & 78,47 & $76,83-80,03$ & 21,53 & $19,97-23,17$ \\
\hline Fundamental completo / Médio incompleto & & 83,14 & $80,28-85,66$ & 16,86 & $14,34-19,72$ \\
\hline Médio Completo e mais & & 83,34 & $81,64-84.91$ & 16,66 & $15,10-18,36$ \\
\hline Cor da pele & 8.532 & & & & \\
\hline Branca e outras & & 80,06 & $78,32-81,69$ & 19,94 & $18,31-21,68$ \\
\hline Preta e parda & & 82,74 & $81,41-83,99$ & 17,26 & $16,01-18,59$ \\
\hline Região & 8.534 & & & & \\
\hline Norte & & 83,78 & $82,08-85,34$ & 16,22 & $14,66-17,92$ \\
\hline Nordeste & & 80,21 & $78,71-81,63$ & 19,79 & $18,37-21,29$ \\
\hline Sudeste & & 82,13 & $80,05-84,04$ & 17,87 & $15,96-19,95$ \\
\hline Sul & & 79,98 & $77,36-82,37$ & 20,02 & $17,63-22,64$ \\
\hline Centro-Oeste & & 82,23 & $79,5-84,59$ & 17,77 & $15,41-20,41$ \\
\hline Índice de massa corporal & 8.441 & & & & \\
\hline Baixo/Normal & & 86,19 & $84,69-87,57$ & 13,81 & $12,43-15,31$ \\
\hline Sobrepeso & & 78,90 & $77,04-80,66$ & 21,10 & $19,34-22,96$ \\
\hline Obesidade & & 76,70 & $74,07-79,14$ & 23,30 & $20,86-25,93$ \\
\hline Consumo de carne vermelha com gordura & 8.054 & & & & \\
\hline $\operatorname{Sim}$ & & 82,03 & $79,93-83,96$ & 17,97 & $16,04-20,07$ \\
\hline Não & & 81,06 & $79,73-82,33$ & 18,94 & $17,60-20,27$ \\
\hline Consumo de bebida alcoólica & 8.534 & & & & \\
\hline Leve/Moderado & & 82,05 & $79,26-84,54$ & 17,95 & $15,46-20,74$ \\
\hline Abusivo & & 84,16 & $79,99-87,59$ & 15,84 & $12,41-20,01$ \\
\hline Não & & 81,04 & $79,80-82,21$ & 18,96 & $17,79-20,20$ \\
\hline
\end{tabular}

continua

Este estudo apresentou como limitações: a impossibilidade de atestar relação causal e a possibilidade de causalidade reversa. Por um lado, houve a impossibilidade de atestar a relação causal entre as variáveis examinadas por tratar-se de estudo transversal. Pelo fato do desfecho e as suas causas serem analisadas em momento único, as associações aqui descritas podem ser resultados de mudanças de estilos de vida e outras modificações referentes ao tratamento. Por outro lado, cabe mencionar a possibilidade de causalidade reversa entre as DCNT aqui estudadas e o LDLColesterol aumentado. Assim, esses resultados devem ser interpretados com prudência. Contudo, para controle desse viés, as análises metodológicas foram feitas de forma critériosa e tentou-se controlar tal situação por meio de modelo multivariado, seguindo a hierarquia de blocos de determinação causal com embasamento na literatura científica ${ }^{15,16,19-22,37}$.

O aumento do LDL-Colesterol nos adultos a partir dos 30 anos, identificado nesta pesqui- 
Tabela 1. Prevalência de LDL-Colesterol limiar ótimo (<130 mg/dl) e aumentado ( $\geq 130 \mathrm{mg} / \mathrm{dL})$ e IC95\% em adultos ( $\geq 18$ anos), segundo variáveis sociodemográficas, antropométricas, estilo de vida, doenças crônicas não transmissíveis e autoavaliação de saúde. Pesquisa Nacional de Saúde, Brasil, 2014-2015.

\begin{tabular}{|c|c|c|c|c|c|}
\hline LDL-Colesterol & & & imiar ótimo & & Aumentado \\
\hline Variáveis & $\mathbf{n}^{*}$ & $\%$ & IC $95 \%$ & $\%$ & IC $95 \%$ \\
\hline Tabagismo & 8.527 & & & & \\
\hline Sim & & 79,75 & $76,60-82,58$ & 20,25 & $17,42-23,40$ \\
\hline Não & & 81,69 & $80,53-82,79$ & 18,31 & $17,21-19,47$ \\
\hline Anemia & 7.917 & & & & \\
\hline Sim & & 86,71 & $84,06-88,97$ & 13,29 & $11,03-15,94$ \\
\hline Não & & 80,62 & $79,40-81,79$ & 19,38 & $18,21-20,60$ \\
\hline Hipertensão arterial & 8.157 & & & & \\
\hline Sim & & 76,73 & $74,35-78,96$ & 23,27 & $21,04-25,65$ \\
\hline Não & & 82,61 & $81,35-83,80$ & 17,39 & $16,20-18,65$ \\
\hline Insuficiência renal & 8.534 & & & & \\
\hline Sim & & 77,33 & $72,71-81,36$ & 22,67 & $18,64-27,29$ \\
\hline Não & & 81,66 & $80,55-82,73$ & 18,34 & $17,27-19,45$ \\
\hline Diabetes & 8.306 & & & & \\
\hline Sim & & 75,28 & $71,53-78,68$ & 24,72 & $21,32-28,47$ \\
\hline Não & & 82,03 & $80,88-83,12$ & 17,97 & $16,88-19,12$ \\
\hline Autoavaliação de saúde & 8.527 & & & & \\
\hline Muito Bom/Bom & & 83,46 & $82,12-84,73$ & 16,54 & $15,27-17,88$ \\
\hline Regular & & 77,95 & $75,90-79,87$ & 22,05 & $20,13-24,10$ \\
\hline Muito ruim/Ruim & & 75,76 & $71,47-79,59$ & 24,24 & $20,41-28,53$ \\
\hline
\end{tabular}

${ }^{\star} \mathrm{O}$ valor total da amostra é 8.534 participantes, porém dados faltantes não foram apresentados. IC $95 \%$ Intervalo de $95 \%$ de confiança.

sa, pode ser explicado pelas alterações lipídicas decorrentes do processo de envelhecimento gradativo, pois a chance aumenta com o avanço da idade. Os mecanismos de envelhecimento acometem os tecidos e os órgãos, resultando em alterações no endotélio hepático, aumento da resistência à insulina, diminuição do androgênio nos homens e dos hormônios nas mulheres decorrentes da menopausa e pós-climatério ${ }^{38}$. Resultados semelhantes também foram encontrados em outras pesquisas no $\mathrm{Brasil}^{23,43} \mathrm{e}$ em outros países, como China ${ }^{44}$ e Estados Unidos ${ }^{45}$.

A população com IMC alterado apresentou maiores prevalências de LDL-Colesterol aumentado, e esses dados confirmam que o sobrepeso e a obesidade, principalmente a adiposidade abdominal, contribuem para a ocorrência de dislipidemias $^{39}$. Na obesidade, a resistência à insulina é o distúrbio metabólico mais comum e está relacionada ao aumento do colesterol; isso, devido a elevações dos níveis de ácidos graxos livres (AGL), que culmina com a redução da degradação da ApoB100, que é componente principal das lipoproteínas de muito baixa densidade (VLDL) e a maior secreção hepática de VLDL. Desse pro- cesso, tem-se como consequência elevações de TG (o seu excesso é secretado como VLDL), levando à hipertrigliceridemia. Além disso, ocorre a metabolização de VLDL em partículas pequenas e densas de LDL-Colesterol, gerando o seu acúmulo $^{39}$.

Residir na Região Nordeste foi fator de risco para LDL-Colesterol aumentado. Uma possível explicação para isso seriam os vazios assistenciais evidenciados por menores prevalências de consultas médicas referidas nos últimos 12 meses, o que contribui para o subdiagnóstico e tratamento tardio, principalmente nas regiões Nordeste e Norte, se comparadas às outras regiões ${ }^{46}$. Outra hipótese é o aumento dos fatores de risco, como a obesidade, que apresentou tendência de crescimento nos últimos 11 anos $^{47}$. Apesar dos dados da PNS mostrarem melhorias e avanços no acesso e uso dos serviços de saúde, diferenças regionais ainda são observadas no país ${ }^{46}$. Todas as explicações carecem de evidências empíricas e teóricas; portanto, necessitam ser melhor investigadas.

Indivíduos com anemia apresentaram menor prevalência de LDL-Colesterol aumentado. Bai- 
Tabela 2. Fatores associados ao LDL-Colesterol aumentado ( $\geq 130 \mathrm{mg} / \mathrm{dL})$ em adultos $\geq 18$ anos, razões de prevalência bruta (modelo de regressão 1) e ajustada (modelo de regressão 2) e seus respectivos IC95\%, segundo as variáveis selecionadas. Pesquisa Nacional de Saúde, Brasil, 2014-2015.

\begin{tabular}{|c|c|c|c|c|c|c|}
\hline Variável & $\mathbf{R P b}$ & IC95\% & $\mathbf{p}$ & RPaj $^{\mathbf{a}}$ & IC95\% & $\mathbf{p}$ \\
\hline \multicolumn{7}{|l|}{ Sexo } \\
\hline Masculino & 1 & & & 1 & & \\
\hline Feminino & 1,16 & $1,03-1,30$ & 0,013 & 1,16 & $1,03-1,30$ & 0,011 \\
\hline \multicolumn{7}{|l|}{ Faixa etária em anos } \\
\hline 18 a 29 & 1 & & & 1 & & \\
\hline 30 a 44 & 1,99 & $1,60-2,49$ & $<0,01$ & 1,98 & $1,58-2,48$ & $<0,01$ \\
\hline 45 a 59 & 2,90 & $2,34-3,59$ & $<0,01$ & 2,87 & $2,31-3,56$ & $<0,01$ \\
\hline 60 anos ou mais & 2,78 & $2,24-3,46$ & $<0,01$ & 2,72 & $2,17-3,41$ & $<0,01$ \\
\hline \multicolumn{7}{|l|}{ Escolaridade } \\
\hline Analfabeto/Fundamental incompleto & 1 & & & 1 & & \\
\hline Fundamental completo/Médio incompleto & 0,78 & $0,66-0,93$ & 0,006 & 0,78 & $0,66-0,93$ & 0,007 \\
\hline Médio Completo e mais & 0,77 & $0,68-0,88$ & $<0,01$ & 0,75 & $0,66-0,85$ & $<0,01$ \\
\hline \multicolumn{7}{|l|}{ Raça/cor da pele } \\
\hline Branca e outras & 1 & & & 1 & & \\
\hline Preta e parda & 0,87 & $0,77-0,97$ & 0,012 & 0,82 & $0,73-0,93$ & 0,003 \\
\hline \multicolumn{7}{|l|}{ Região } \\
\hline Norte & 1 & & & 1 & & \\
\hline Nordeste & 1,22 & $1,08-1,38$ & 0,002 & 1,18 & $1,05-1,34$ & 0,007 \\
\hline Sudeste & 1,10 & $0,95-1,28$ & 0,208 & 1,05 & $0,90-1,22$ & 0,49 \\
\hline Sul & 1,23 & $1,05-1,45$ & 0,01 & 1,12 & $0,94-1,33$ & 0,199 \\
\hline Centro-Oeste & 1,09 & $0,92-1,30$ & 0,3 & 1,07 & $0,90-1,27$ & 0,412 \\
\hline \multicolumn{7}{|l|}{ Índice de Massa Corporal } \\
\hline Baixo/Normal & 1 & & & 1 & & \\
\hline Sobrepeso & 1,53 & $1,34-1,75$ & $<0,01$ & 1,51 & $1,32-1,73$ & $<0,01$ \\
\hline Obesidade & 1,69 & $1,45-1,96$ & $<0,01$ & 1,63 & $1,41-1,91$ & $<0,01$ \\
\hline \multicolumn{7}{|l|}{ Consumo de carne vermelha com gordura } \\
\hline Não & 1 & & & 1 & & \\
\hline Sim & 0,95 & $0,83-1,08$ & 0,433 & 0,97 & $0,85-1,11$ & 0,688 \\
\hline \multicolumn{7}{|l|}{ Consumo de bebida alcoólica } \\
\hline Não & 1 & & & 1 & & \\
\hline Leve/Moderado & 0,95 & $0,81-1,11$ & 0,501 & 1,01 & $0,86-1,19$ & 0,897 \\
\hline Abusivo & 0,84 & $0,65-1,07$ & 0,154 & 0,87 & $0,70-1,12$ & 0,291 \\
\hline \multicolumn{7}{|l|}{ Tabagismo } \\
\hline Não & 1 & & & 1 & & \\
\hline Sim & 1,10 & $0,94-1,29$ & 0,218 & 1,09 & $0,92-1,28$ & 0,282 \\
\hline \multicolumn{7}{|l|}{ Anemia } \\
\hline Não & 1 & & & 1 & & \\
\hline Sim & 0,69 & $0,56-0,83$ & $<0,01$ & 0,66 & $0,54-0,80$ & $<0,01$ \\
\hline \multicolumn{7}{|l|}{ Hipertensão arterial } \\
\hline Não & 1 & & & 1 & & \\
\hline Sim & 1,34 & $1,18-1,51$ & $<0,01$ & 1,25 & $1,10-1,42$ & $<0,01$ \\
\hline \multicolumn{7}{|l|}{ Insuficiência Renal } \\
\hline Não & 1 & & & 1 & & \\
\hline Sim & 1,24 & $1,01-1,51$ & 0,037 & 1,11 & $0,91-1,37$ & 0,287 \\
\hline \multicolumn{7}{|l|}{ Diabetes } \\
\hline Não & 1 & & & 1 & & \\
\hline Sim & 1,38 & $1,18-1,61$ & $<0,01$ & 1,29 & $1,10-1,50$ & 0,001 \\
\hline
\end{tabular}


Tabela 2. Fatores associados ao LDL-Colesterol aumentado ( $\geq 130 \mathrm{mg} / \mathrm{dL})$ em adultos $\geq 18$ anos, razões de prevalência bruta (modelo de regressão 1) e ajustada (modelo de regressão 2) e seus respectivos IC95\%, segundo as variáveis selecionadas. Pesquisa Nacional de Saúde, Brasil, 2014-2015.

\begin{tabular}{lrrrrrr}
\hline \multicolumn{1}{c}{ Variável } & RPb & IC95\% & p & RPaj $^{\mathbf{a}}$ & IC95\% & p \\
\hline Autoavaliação de saúde & & & & & & \\
$\quad$ Muito Bom/Bom & 1 & & & 1 & & \\
Regular & 1,33 & $1,18-1,50$ & $<0,01$ & 1,26 & $1,11-1,43$ & $<0,01$ \\
Muito ruim/Ruim & 1,47 & $1,22-1,76$ & $<0,01$ & 1,32 & $1,09-1,60$ & 0,004 \\
\hline
\end{tabular}

$\mathrm{RPb}$ : Razão de prevalência bruta (modelo de regressão 1); RPaja: Razão de prevalência ajustada (modelo de regressão 2) ajustado por sexo, escolaridade, cor de pele e região; IC95\%: Intervalo de 95\% de confiança.

Tabela 3. Fatores associados ao LDL-Colesterol aumentado ( $\geq 130 \mathrm{mg} / \mathrm{dL}$ ) em adultos $\geq 18$ anos, razão de prevalência ajustada (modelo de regressão 3) e seus respectivos IC95\%, segundo as variáveis selecionadas. Pesquisa Nacional de Saúde, Brasil, 2014-2015.

\begin{tabular}{lrrr}
\hline \multicolumn{1}{c}{ Variável } & RPaj $^{\mathbf{b}}$ & IC95\% & p \\
\hline Sexo & & & \\
$\quad$ Masculino & 1 & & \\
$\quad$ Feminino & 1,08 & $0,96-1,22$ & 0,177 \\
Faixa etária em anos & & & \\
$\quad 18$ a 29 & 1 & & \\
30 a 44 & 1,99 & $1,58-2,54$ & $<0,01$ \\
45 a 59 & 2,89 & $2,29-3,64$ & $<0,01$ \\
60 anos ou mais & 2,90 & $2,29-3,68$ & $<0,01$ \\
Região & & & \\
$\quad$ Norte & 1 & & \\
Nordeste & 1,16 & $1,02-1,32$ & 0,028 \\
Sudeste & 0,94 & $0,80-1,10$ & 0,474 \\
Sul & 1,06 & $0,89-1,25$ & 0,5 \\
Centro-Oeste & 0,97 & $0,82-1,16$ & 0,762 \\
Índice de Massa Corporal & & & \\
Baixo/Normal & 1 & & \\
Sobrepeso & 1,32 & $1,15-1,51$ & $<0,01$ \\
Obesidade & 1,41 & $1,19-1,65$ & $<0,01$ \\
Anemia & & & \\
Não & 1 & & \\
Sim & 0,66 & $0,54-0,80$ & $<0,01$ \\
\hline
\end{tabular}

RPaj: Razão de prevalência ajustada (modelo de regressão 3/ modelo final) ajustado por todas as variáveis explicativas com valor de $\mathrm{p}<0,20$ na análise bivariada (RPb); IC95\%: Intervalo de $95 \%$ de confiança.

xos valores plasmáticos de colesterol são descritos em diversos tipos de anemias adquiridas e hereditárias (megaloblástica, ferropriva, aplástica, associada à doença hepática, esferocitose hereditária, falciforme e talassemias $)^{40}$; isso, devido à maior utilização do colesterol plasmático, determinado pela renovação de lípides eritrocitários nos casos de menor sobrevida ou hemólise aumentada e a maior diluição do colesterol sérico decorrente do aumento do volume plasmático secundário aos baixos valores de hematócrito e hemoglobina ${ }^{40}$. $\mathrm{Na}$ anemia falciforme e em talassemias, a disfunção hepática reduz a produção endógena de colesterol e amplifica as alterações no perfil lipídico, podendo os portadores apresentar baixos valores de CT, LDL e HDL ${ }^{40}$.

O sexo feminino apresentou-se como fator de risco nas análises bivariada e ajustada. A diferença entre o sexo e a prevalência de dislipidemias não está bem estabelecida na literatura ${ }^{19}$. Entretanto, documenta-se a alta prevalência das dislipidemias nas mulheres durante a menopausa ${ }^{48}$ e no pós-climatério ${ }^{8}$, provavelmente pela perda de proteção hormonal que ocorre nessas fases da vida $^{49}$.

A cor de pele preta e parda mostrou-se fator protetor nas análises bivariada e ajustada, em concordância com resultados do estudo Elsa -Brasil e outras pesquisas realizadas nos Estados Unidos $^{50,51}$, que indicaram menor prevalência de dislipidemia entre negros ${ }^{52}$. Possível motivo para esse achado deve-se ao fato de que o status socioeconômico, padrão alimentar e outros fatores ambientes diferem-se amplamente por etnias ${ }^{51}$, contudo novas pesquisas são necessárias para maior exploração da temática no Brasil.

Quanto à escolaridade, as análises bivariada e ajustada mostraram que os indivíduos com menor escolaridade apresentaram maiores prevalências de LDL-Colesterol aumentado, ressalta-se que os fatores de risco cardiovascular são mais prevalentes entre indivíduos com baixo status socioeconômico, que inclui a escolaridade, o que pode ser decorrente do maior acesso a serviços de saúde e práticas de promoção em população com maior escolaridade ${ }^{53}$. 
Doenças como hipertensão arterial e diabetes mostraram-se como fatores de risco para LDLColesterol, mas perderam a significância estatística no modelo final, devido ao efeito do ajuste pela idade. Destaca-se que essas variáveis são mais frequentes entre idosos, o que poderia justificar esses resultados. Ainda, cabe ressaltar que as DCNT aqui estudadas cursam com elevações de níveis de LDL pelos próprios mecanismos fisiopatológicos dessas doenças ${ }^{8,41,42}$. É documentada a relação entre as DCNT e as dislipidemias, bem como que essas doenças cursam com risco aumentado de DCV ateroesclerótica ${ }^{8,41,42}$. Na insuficiência renal, ocorrem alterações no metabolismo das lipoproteínas por atividade inadequada de enzimas e vias metabólicas que resultam em dislipidemia ${ }^{41}$. No diabetes, a dislipidemia é uma anormalidade metabólica comum, em razão de fatores como a deficiência de insulina ou resistência a ela, adipocitocinas e a hiperglicemia que, quando crônica, resulta em maior acúmulo de partículas densas de $\mathrm{LDL}^{42}$. Na hipertensão, a agressão ao endotélio vascular causa a disfunção endotelial, culminado no aumento da permeabilidade das lipoproteínas plasmáticas e favorecendo a retenção de partículas de $\mathrm{LDL}^{8}$.

No bloco de estilo de vida, nenhuma das variáveis manteve-se nos modelos 2 e 3 , entretanto são bem estabelecidas as evidências da importância do padrão alimentar e da adoção de estilo de vida saudável como medidas de controle e prevenção das dislipidemias ${ }^{8,54,55}$. Recomenda-se a redução de açúcares e a inclusão de carnes magras, frutas, grãos e hortaliças na dieta $^{8}$. A prática de exercícios físicos reduz morbimortalidade cardiovascular pelo aprimoramento do funcionamento da HDL, aumento da resistência à oxidação da LDL e aumento do fluxo de colesterol ${ }^{8}$. O consumo de bebida alcoólica não é recomen- dado para indivíduos com hipertrigliceridemia, devido à combinação do etanol e ácidos graxos saturados potencializarem a elevação dos $\mathrm{TG}^{8}$, já o consumo moderado de álcool (aceitável o consumo $\leq 10 \mathrm{~g} / \mathrm{dia}$ - 1 unidade) aumenta a concentração plasmática de HDL e diminui as concentrações de LDL, sendo associado à redução do risco de $\mathrm{DCV}^{55}$. Quanto ao tabagismo, o ato de fumar, além de lesar o endotélio arterial, também aumenta os níveis de CT e LDL e diminui HDL, assim, a cessação de fumar é benéfica em qualquer fase da vida ${ }^{8,55}$.

Os dados deste estudo também apontam para a relevância da autoavaliação de saúde. Essa variável é considerada um preditor de morbimortalidade $^{2}$ e, por isso, a importância de ser estudada. Os resultados aqui apresentados foram semelhantes à pesquisa com dados da PNS, que mostrou forte associação entre a autoavaliação e as dislipidemias para os que apresentavam avaliação de seu estado de saúde ruim ${ }^{19}$.

O LDL-Colesterol aumentado na população brasileira está associado com o envelhecimento, sobrepeso, obesidade, morar na região Nordeste e ter anemia. Esses dados reforçam a importância tanto do monitoramento do perfil lipídico em adultos - devido às elevações dos níveis LDL, em função do envelhecimento e alterações de IMC - quanto para o diagnóstico em locais de menor acesso para a população brasileira. Além disso, o Brasil possui diferenças regionais, culturais e socioeconômicas; assim, torna-se fundamental conhecer tais características para identificar e enfrentar as iniquidades em saúde. Ademais, este estudo reforça a importância de ações de controle e prevenção de dislipidemias, como a adoção de medidas de estilos de vida saudável, como dieta e manutenção do IMC dentro do preconizado nos adultos brasileiros. 


\section{Colaboradores}

ACMGN Sá participou da concepção do estudo, planejamento, delineamento, análises estatísticas, interpretação dos dados, redação do artigo, elaborou a primeira versão do manuscrito e aprovou a versão a ser publicada. IE Machado participou do planejamento, análises estatísticas e revisão crítica do artigo. RTI Bernal participou das análises estatísticas e interpretação dos dados. DC Malta participou da concepção e planejamento do estudo laboratorial da PNS, concepção, interpretação dos dados, revisão crítica e aprovou a versão final do manuscrito.

\section{Agradecimentos}

Ao Conselho da Coordenação de Aperfeiçoamento de Pessoal de Nível Superior (CAPES), pela bolsa de Doutorado recebida pela autora ACMGNS, e ao Conselho Nacional de Desenvolvimento Científico e Tecnólógico (CNPq), pela bolsa de Produtividade em Pesquisa recebida pela autora DCM. À Secretaria de Vigilância em Saúde do MS.

\section{Referências}

1. Kannel WB, Castelli WP, Gordon T. Cholesterol in the prediction of atherosclerotic disease. New perspectives based on the Framingham study. Ann Intern Med 1979; 90(1):85-91.

2. Nayor M, Vasan RS. Recent Update to th Cholesterol Treatment Guidelines: A Comparison With International Guidelines. Circulation 2016; 133(18):17951806.

3. Diaz MN, Frei B, Vita JA, Keaney Junior JF. Antioxidants and atherosclerotic heart disease. $N$ Engl J Med 1997; 37(6):408-416.

4. Coelho VG, Caetano LF, Liberatori Júnior RDR, Cordeiro JA, Souza DRS. Perfil Lipídico e Fatores de Risco para Doenças Cardiovasculares em Estudantes de Medicina. Arq Bras Cardiol 2005; 85(1):57-62.

5. Durrington P. Dyslipidaemia. Lancet 2003; 362(9385):717-731.

6. Cholesterol Treatment Trialists' (CTT) Collaboration. Efficacy and safety of more intensive lowering of LDL cholesterol: a meta-analysis of data from 170,000 participants in 26 randomised trials. Lancet 2010; 376(9753):1670-1681.

7. Cholesterol Treatment Trialists' (CTT) Collaboration. The effects of lowering LDL cholesterol with statin therapy in people at low risk of vascular disease: meta-analysis of individual data from 27 randomised trials. Lancet 2012; 380(9841):581-590.

8. Faludi AA, Izar MCO, Saraiva JFK, Chacra APM, Bianco HT, Afiune A Neto, Bertolami A, Pereira AC, Lottenberg AM, Sposito AC, Chagas ACP, Casella A Filho, Simão AF, Alencar AC Filho, Caramelli B, Magalhães CC, Negrão CE, Ferreira CEDS, Scherr C, Feio CMA, Kovacs C, Araújo DB, Magnoni D, Calderaro D, Gualandro DM, Mello EP Junior, Alexandre ERG, Sato EI, Moriguchi EH, Rached FH, Santos FCD, Cesena FHY, Fonseca FAH, Fonseca HARD, Xavier HT, Mota ICP, Giuliano ICB, Issa JS, Diament J, Pesquero JB, Santos JED, Faria JR Neto, Melo JX Filho, Kato JT, Torres KP, Bertolami MC, Assad MHV, Miname MH, Scartezini M, Forti NA, Coelho OR, Maranhão RC, Santos RDD Filho, Alves RJ, Cassani RL, Betti RTB, Carvalho T, Martinez TLDR, Giraldez VZR, Salgado W Filho. Atualização da Diretriz Brasileira de Dislipidemias e Prevenção da Aterosclerose - 2017. Arq Bras Cardiol 2017; 109(2 Supl. 1):176.

9. Ribeiro AL, Ducan BB, Bramt LCC, Lotudo PA, Mill JG, Barreto SM. Cardiovascular Health in Brazil: Trends and Perspectives. Circulation 2016; 133(4):422-433.

10. Upadhyay RK. Emerging risk biomarkers in cardiovascular diseases and disorders. J Lipids 2015; 2015:971453.

11. World Health Organization (WHO). Global Health Risks: Mortality and burden of disease attributable to selected major risks. Geneva: WHO; 2009.

12. Mattiuzzi C, Sanchis-Gomar F, Lippi G. Worldwide burden of LDL cholesterol: Implications in cardiovascular disease. Nutr Metab Cardiovasc Dis 2020; 30(2):241-244.

13. Institute for Health Metrics and Evaluation (IHME). GBD Compare Data Visualization. Seattle: IHME; 2017. 
14. Zhong Z, Liu J, Li B, Li C, Liu Z, Yang M, Zhong W, Zhao P. Serum lipid profiles in patients with acute myocardial infarction in Hakka population in Southern China. Lipids Health Dis 2017; 16(1):246.

15. Bayram F, Kocer D, Gundogan K, Kaya A, Demir O, Coskun R, Sabuncu T, Karaman A, Cesur M, Rizzo M. Prevalence of dyslipidemia and associated risk factors in Turkish adults. J Clin Lipidol 2014; 8(2):206-216.

16. Opoku S, Gan Y, Fu W, Chen D, Addo-Yobo E, Trofimovitch D, Yue W, Yan F, Wang Z, Lu Z. Prevalence and risk factors for dyslipidemia among adults in rural and urban China: findings from the China National Stroke Screening and prevention project (CNSSPP). BMC Public Health 2019; 19:1500.

17. Musunuru K. Atherogenic Dyslipidemia: Cardiovascular Risk and Dietary Intervention. Lipids 2010; 45(10):907-914.

18. Knight BS, Sunn N, Pennell CE, Adamson SL, Lye SJ. Developmental regulation of cardiovascular function is dependent on both genotype and environment. Am J Physiol Heart Circ Physiol 2009; 297(6):H2234-2241.

19. Pereira LPP, Sichieri PR, Segri NJ, Silva RMVG, Ferreira MG. Dislipidemia autorreferida na região Centro-Oeste do Brasil: prevalência e fatores associados. Cien Saude Colet 2015; 20(6):1815-1824.

20. Wang S, Xu L, Joanas JB, You QS, Wang YX, Yang H. Prevalence and associated factors of dyslipidemia in the adult Chinese population. PLoS One 2011; 6(3):e17326.

21. Moraes SA, Checchio MV, Freitas ICM. Dislipidemia e fatores associados em adultos residentes em Ribeirão Preto, SP. Resultados do Projeto EPIDCV. Arq Bras Endocrinol Metab 2013; 57(9):691-701.

22. Qi L, Ding X, Tang W, Li Q, Mao D, Wang Y. Prevalence and Risk Factors Associated with Dyslipidemia in Chongqing, China. Int J Environ Res Public Health 2015; 12(10):13455-13465.

23. Lotufo PA, Santos RD, Sposito AC, Bertolami M, Rocha-Faria J Neto, Izar MC, Szwarcwald C, Prado RR, Stoppa SR, Malta DC, Bensenor IM. Prevalência de Diagnóstico Médico de Colesterol Alto Autorreferido na População Brasileira: Análise da Pesquisa Nacional de Saúde, 2013. Arq Bras Cardiol 2017; 108(5):411-416.

24. Souza-Júnior PRB, Freitas MPS, Antonaci GA, Szwarcwald CL. Desenho da amostra da Pesquisa Nacional de Saúde 2013. Epidemiol Serv Saúde 2015; 24(2):207216.

25. Szwarcwald CL, Malta DC, Azevedo C, Souza-Júnior PRB, Rosenfeld LG. Exames laboratoriais da pesquisa nacional de saúde: Metodologia de amostragem, coleta, e análise dos dados. Rev Bras Epidemiol 2019; 22(Supl. 2):1-9.

26. Malta DC, Szwarcwald CL, Machado IE, Pereira CA, Figueiredo AW, Sá ACMGN, Velasquez-Melendez G, Santos FM, Souza-Júnior PRB, Stopa SR, Rosenfeld LG. Prevalência de colesterol total e frações alterados na população adulta brasileira: Pesquisa Nacional de Saúde. Rev Bras Epidemiol 2019; 22(Supl. 2):1-13.

27. Ridker PM. LDL cholesterol: controversies and future therapeutic directions. Lancet 2014; 384(9943):607617.
28. Instituto Brasileiro de Geografia e Estatística (IBGE). Pesquisa Nacional de Saúde 2013: percepção do estado de saúde, estilos de vida e doenças crônicas: Brasil, Grandes Regiões e Unidades da Federação. Rio de Janeiro: IBGE; 2014.

29. Instituto Brasileiro de Geografia e Estatística (IBGE). Pesquisa Nacional de Saúde 2013: Manual de Entrevista. Rio de Janeiro: IBGE; 2013.

30. Expert Panel on Detection, Evaluation, and Treatment of High Blood Cholesterol in Adults. Executive summary of the third report of the National Cholesterol Education Program (NCEP) expert panel on detection, evaluation, and treatment of high blood cholesterol in adults (Adult Treatment Panel III). JAMA 2001; 285(19):2486-2497.

31. World Health Organization (WHO). Obesity: preventing and managing the global epidemic. Geneva: WHO; 2000.

32. Centers for Disease Control and Prevention (CDC). Fact Sheets - Preventing Excessive Alcohol Use. Atlanta: CDC; 2020.

33. Kidney Disease Improving Global Outcomes. KDIGO 2012 Clinical Practice Guideline for the Evaluation and Management of Chronic Kidney Disease. Kidney Int Suppl 2013; 3(1):1-150.

34. World Health Organization (WHO). Use of glycated haemoglobin (HbAlc) in the diagnosis of diabetes mellitus. Genebra: WHO; 2011.

35. American Diabetes Association. 2. Classification and Diagnosis of Diabetes: Standards of Medical Care in Diabetes-2018. Diabetes Care 2018; 41 (Supl. 1):S13-S27.

36. Malachias MVB, Souza WKSB, Plavnik FL, Rodrigues CIS, Brandão AA, Neves MFT, Bortolotto LA, Franco RJS, Poli-de-Figueiredo CE, Jardim PCBV, Amodeo C, Barbosa ECD, Koch V, Gomes MAM, Paula RB, Póvoa RMS, Colombo FC, Ferreira Filho S, Miranda RD, Machado CA, Nobre F, Nogueira AR, Mion Júnior D, Kaiser S, Forjaz CLM, Almeida FA, Martim JFV, Sass N, Drager LF, Muxfeldt E, Bodanese LC, Feitosa AD, Malta D, Fuchs S, Magalhães ME, Oigman W, Moreira Filho O, Pierin AMG, Feitosa GS, Bortolotto MRFL, Magalhães LBNC, Silva ACS, Ribeiro JM, Borelli FAO, Gus M, Passarelli Júnior O, Toledo JY, Salles GF, Martins LC, Jardim TSV, Guimarães ICB, Antonello IC, Lima Júnior E, Matsudo V, Silva GV, Costa LS, Alessi A, Scala LCN, Coelho EB, Souza D, Lopes HF, Gowdak MMG, Cordeiro Júnior AC, Torloni MR, Klein MRST, Nogueira PK, Lotaif LAD, Rosito GBA, Moreno Júnior H. 7a Diretriz Brasileira de Hipertensão Arterial: Capítulo 2 - Diagnóstico e Classificação. Arq. Bras. Cardiol. 2016; 107(3 Supl. 3):1-83.

37. Bergmann MLA, Bergmann GG, Halpern R, Rech RR, Constanzi CB, Alli LR. Colesterol Total e Fatores Associados: Estudo de Base Escolar no Sul do Brasil. Arq Bras Cardiol 2011; 97(1):17-25.

38. Liu HH, Li JJ. Aging and dyslipidemia: a review of potential mechanisms. Ageing Res Rev 2015; 19:43-52.

39. Vekic J, Zelikovic A, Stefanovic A, Jelic-Ivanovic Z, Spasojevic-Kalimanovska V. Obesity and dyslipidemia. Metabolism 2019; 92:71-81. 
40. Naoum FA. Alterações do perfil lipídico nas anemias. Rev Bras Hematol Hemoter 2005; 27(3):223-226.

41. Mikolasevic I, Žutelija M, Mavrinac V, Orlic L. Dyslipidemia in patients with chronic kidney disease: etiology and management. Int $J$ Nephorol Renovasc Dis 2017; 10:35-45.

42. Schofield JD, Liu Y, Rao-Balakrishna P, Malik RA, Soran H. Dyslipidemia. Diabetes Ther 2016; 7(2):203219.

43. Brasil. Ministério da Saúde (MS). Vigitel Brasil 2014: vigilância dos fatores de risco e proteção para as doenças crônicas por inquérito telefônico. Brasília: MS; 2015.

44. Zhang M, Deng Q, Wang L, Huang Z, Zhou M, Li Y, Zhao Z, Zhang Y, Wang L. Prevalence of dyslipidemia and achievement of low-density lipoprotein cholesterol targets in Chinese adults: A nationally representative survey of 163,641 adults. Int J Cardiol 2018; 260:196-203.

45. Tóth PP, Potter D, Ming EE. Prevalence of lipid abnormalities in the United States: the National Health and Nutrition Examination Survey 2003-2006. J Clin Lipidol 2012; 6(4):325-330.

46. Stopa SR, Malta DC, Monteiro CN, Szwarcwald CL, Goldbaum M, Cesar CLG. Acesso e uso de serviços de saúde pela população brasileira, Pesquisa Nacional de Saúde 2013. Rev Saude Publica 2017; 51 (Supl. 1):3s.

47. Malta DC, Silva AG, Tonaco LAB, Freitas MIF, Velasquez-Melendez G. Tendência temporal da prevalência de obesidade mórbida na população adulta brasileira entre os anos de 2006 e 2017. Cad Saude Publica 2019; 35(9):e00223518.

48. Phan BA, Toth PP. Dyslipidemia in women: etiology and management. Int J Womens Health 2014; 6:185194.

49. Edmunds E, Lip GYH. Cardiovascular risk in women: the cardiologist perspective. Q J Med 2000; 93(3):135145.

50. Frank AT, Zhao B, Jose PO, Azar KM, Fortmann SP, Palaniappan LP. Racial/ethnic differences in dyslipidemia patterns. Circulation 2014; 129(5):570-579.

51. McIntosh MS, Kumar V, Kalynych C, Lott M, Hsi A, Chang JL, Lerman R. Racial Differences in Blood Lipids Lead to Underestimation of Cardiovascular Risk in Black Women in a Nested observational Study. Glob Adv Health Med 2013; 2(2):76-79.

52. Santos RD, Bensenor IM, Pereira AC, Lotufo PA. Dyslipidemia according to gender and race: The Brazilian Longitudinal Study of Adult Health (ELSA-Brasil). J Clin Lipidol 2016; 10(6):1362-1368.

53. Malta DC, Bernal RTI, Lima MG, Araújo SSC, Silva MMAD, Freitas MIF, Barros MBA. Doenças crônicas não transmissíveis e a utilização de serviços de saúde: análise da Pesquisa Nacional de Saúde no Brasil. Rev Saude Publica 2017; 51 (Supl. 1):4s.
54. Grundy SM, Stone NJ, Bailey AL, Beam C, Birtcher KK, Blumenthal RS, Braun LT, de Ferranti S, FaiellaTommasino J, Forman DE, Goldberg R, Heidenreich PA, Hlatky MA, Jones DW, Lloyd-Jones D, Lopez-Pajares N, Ndumele CE, Orringer CE, Peralta CA, Saseen JJ, Smith SC Jr, Sperling L, Virani SS, Yeboah J. 2018 AHA/ACC/AACVPR/AAPA/ABC/ACPM/ADA/AGS/ APhA/ASPC/NLA/PCNA Guideline on the Management of Blood Cholesterol: Executive Summary: A Report of the American College of Cardiology/American Heart Association Task Force on Clinic Practice Guidelines. J Am Coll Cardiol 2019; 73(24):3168-3209.

55. Mach F, Baigent C, Catapano AL, Koskinas KC, Casula M, Badimon L, Chapman MJ, De Backer GG, Delgado V, Ference BA, Graham IM, Halliday A, Landmesser U, Mihaylova B, Pedersen TR, Riccardi G, Richter DJ, Sabatine MS, Taskinen MR, Tokgozoglu L, Wiklund O, ESC Scientific Document Group. 2019 ESC/ EAS Guidelines for the management of dyslipidaemias: lipid modification to reduce cardiovascular risk. Eur Heart J 2020; 41(1):111-188.

Artigo apresentado em 02/10/2020

Aprovado em 02/10/2020

Versão final apresentada em 04/10/2020

Editores chefes: Maria Cecília de Souza Minayo, Romeu Gomes, Antônio Augusto Moura da Silva 
